\title{
Time-Dependent Propagation of Tsunami-Generated Acoustic-Gravity Waves in the Atmosphere
}

\author{
YUE Wu \\ Department of Mechanical and Aerospace Engineering, University of California, San Diego, La Jolla, California \\ STEFAN G. LLEWELLYN SMITH \\ Department of Mechanical and Aerospace Engineering, and Scripps Institution of Oceanography, \\ University of California, San Diego, La Jolla, California \\ JAMES W. ROTTMAN \\ Department of Mechanical and Aerospace Engineering, University of California, San Diego, La Jolla, California \\ DAVE BROUTMAN \\ Computational Physics, Inc., Springfield, Virginia \\ JEAN-BERNARD H. MINSTER \\ Scripps Institution of Oceanography, University of California, San Diego, La Jolla, California
}

(Manuscript received 24 October 2018, in final form 1 December 2019)

\begin{abstract}
Tsunami-generated linear acoustic-gravity waves in the atmosphere with altitude-dependent vertical stratification and horizontal background winds are studied with the long-term goal of real-time tsunami warning. The initial-value problem is examined using Fourier-Laplace transforms to investigate the time dependence and to compare the cases of anelastic and compressible atmospheres. The approach includes formulating the linear propagation of acoustic-gravity waves in the vertical, solving the vertical displacement of waves and pressure perturbations numerically as a set of coupled ODEs in the Fourier-Laplace domain, and employing den Iseger's algorithm to carry out a fast and accurate numerical inverse Laplace transform. Results are presented for three cases with different atmospheric and tsunami profiles. Horizontal background winds enhance wave advection in the horizontal but hinder the vertical transmission of internal waves through the whole atmosphere. The effect of compressibility is significant. The rescaled vertical displacement of internal waves at $100-\mathrm{km}$ altitude shows an arrival at the early stage of wave development due to the acoustic branch that is not present in the anelastic case. The long-term displacement also shows an $O(1)$ difference between the compressible and anelastic results for the cases with uniform and realistic stratification. Compressibility hence affects both the speed and amplitude of energy transmitted to the upper atmosphere because of fast acoustic waves.
\end{abstract}

\section{Introduction}

Tsunami-generated acoustic-gravity waves propagate in the atmosphere up to the ionosphere, where they have been observed to have an effect on the ionosphere. We simulate the time-dependent propagation of linear acoustic-gravity waves in the atmosphere

Corresponding author: Yue Wu,wuyue@ucsd.edu with altitude-dependent vertical stratification and horizontal background winds.

A typical tsunami propagates along the sea surface and generates internal waves in the atmosphere. Because of their long periods (10-40 min) and small vertical amplitudes in the open ocean $(\leq 0.5 \mathrm{~m})$ compared to their large horizontal wavelength (up to $400 \mathrm{~km}$ ), tsunamis are usually hard to detect by offshore buoys (Artru et al. 2005). Tsunami-generated internal 
waves in the atmosphere are subject to transmission and reflection resulting from background winds and the vertical stratification (Broutman et al. 2014). When waves propagate up to around $300 \mathrm{~km}$ altitude, they have an effect on ionospheric disturbances, which is often quantified by electron density and total electron content (TEC; the total number of electrons present along a path between a transmitter and receiver) and can be detected by the ionospheric sounding techniques using, for example, the global positioning system (GPS). Thus, as first suggested in Peltier and Hines (1976), information carried by atmospheric waves could give tsunami alerts, and this response takes from about $20 \mathrm{~min}$ to a few hours [see, e.g., Fig. 3 of Occhipinti et al. (2008) and Fig. 8 of Occhipinti et al. (2013) for calculations of the propagation time in an incompressible atmosphere].

Artru et al. (2005) first observed tsunami-generated inertia-gravity waves (IGWs) $\left(\mathrm{IGW}_{\text {tsu }}\right)$ with arrival time and direction consistent with the Chile tsunami, while subsequently Occhipinti et al. (2006) showed the existence of IGW $_{\text {tsu }}$ by comparing TEC observations from altimetric satellites (the only instruments measuring the tsunami and $\mathrm{IGW}_{\text {tsu }}$ simultaneously). Since then, researchers have confirmed ionospheric signatures of tsunamis using different methods including GPS data (Rolland et al. 2010; Galvan et al. 2011, 2012; Occhipinti et al. 2013), ionospheric Doppler sounders (Liu et al. 2006), and nightside airglow (Hickey et al. 2010; Occhipinti et al. 2011; Makela et al. 2011). Numerical models (Occhipinti et al. 2006, 2008, 2011; Hickey et al. 2009; Mai and Kiang 2009) have examined the vertical propagation of tsunamigenerated internal waves through the atmosphere. Broutman et al. (2014) investigated the partial reflection of atmospheric waves by including background winds and altitude-dependent stratification. More recent modeling papers include Brissaud et al. (2016), who presented the 2D and 3D finite-difference numerical modeling of the propagation of acousticgravity waves in a stratified viscous atmosphere with attenuation and background winds, Laughman et al. (2017), who presented the finite-volume numerical model of the nonlinear propagation of gravity waves through different tidal wind structures in a vertically varying background, and Rakoto et al. (2017), who extended the seismic normal mode method to the whole solid Earth-ocean-atmosphere system and compared their observations with measurements by DeepOcean Assessment and Reporting of Tsunamis (DART) and GPS-derived TEC signals.

While these recently developed methods allow for very general situations to be modeled, they are, respectively, limited by CFL-type conditions, do not include compressibility, and cannot handle background winds. It is of interest to overcome these limitations and develop rapid algorithms that can be used to study cases with simplified geometry and hence investigate the dominant processes. For example, Wei et al. (2015) examined the time-dependent propagation through a compressible piecewise-constant atmosphere with constant winds and found in particular an early arrival of acoustic waves at high altitude. The linearized equations for a compressible stratified atmosphere possess a dispersion relation that simplifies into gravity and acoustic branches in appropriate limits. The initial disturbance considered by Wei et al. (2015) has nonnegligible content in the acoustic band of the combined Laplace-Fourier transform. There is hence the potential of a first arrival of an acoustic wave front, as seen in Brissaud et al. (2016), who show an acoustic wave front arrival in their Fig. 7; see also Fig. 9.1 of Occhipinti (2016). We aim to study this phenomenon for realistic atmospheric profiles.

Wu et al. (2016a) generalized the two-dimensional algorithm of Broutman et al. (2014) to include compressibility of the atmosphere for realistic profiles. Wu et al. (2016b) included the three-dimensional effects of the steady problem and quantified the dependence of wave energy transmission on the amplitude/direction/altitude of background winds. We extend this approach to study the initial-value problem of the propagation of tsunami-generated linear internal waves in the atmosphere, with the goal of investigating the early-time arrival of acoustic waves. Internal waves have two branches: the gravity branch and the acoustic branch. The two branches are dominated by different mechanisms and propagate at different speeds in the atmosphere. We include time-dependent effects using the Laplace transform with the motivation of investigating the first arrival of acoustic waves and the long-term propagation of gravity waves. We also include vertical gradients of atmospheric stratification and altitudedependent horizontal background winds. Our goal is to simulate wave propagation in a more realistic atmosphere, to quantify the effects of winds and stratification, and to understand how fast and how much energy reaches the lower ionosphere before nonlinearity and ionization become the leading factors in wave propagation at higher altitudes. While the formulation is given for three-dimensional velocities and some preliminary calculations have been carried out in three dimensions in Wu et al. (2016b) and Wu (2018), two-dimensional results are presented here [as was the case in Wei et al. (2015)].

This paper consists of five sections. In section 2, a model formulation for acoustic-gravity waves in a 
compressible atmosphere with altitude-dependent stratification and horizontal background winds is introduced. After a Fourier-Laplace transform, we obtain the rescaled vertical displacement and pressure as solutions to a set of ordinary differential equations (ODEs) in the vertical. In section 3, we compare our numerical method with the analytical study of Wei et al. (2015), and discuss choosing parameters, boundary conditions, integrating the ODEs and recovering solutions in physical space and time. In section 4, we present results for three cases with different atmospheric and tsunami profiles: a two-layer atmosphere with a Gaussianshaped tsunami profile, a uniformly stratified atmosphere with a model tsunami profile, and a realistic atmosphere with a model tsunami profile. Conclusions are given in section 5 .

\section{Formulation}

We set up a Cartesian coordinate in physical space: $x$ and $y$ are the horizontal axes, and $z$ is the vertical axis pointing upward with the origin lying at the average sea surface height. In the time-dependent problem, we treat the tsunami as a traveling disturbance along the $x$ axis at the sea surface with zero displacement at $t=0$.

Following Wu et al. (2016a), we start from the fully compressible Naiver-Stokes equations for an adiabatic, nonrotating, and inviscid fluid. The equations of Occhipinti et al. $(2006,2008,2011)$ correspond to the incompressible limit of these equations. Atmospheric viscosity and attenuation are important for internal waves in the middle and upper atmosphere (Godin 2014) but are beyond the scope of this paper. We have

$$
\begin{aligned}
& \frac{D \mathbf{v}}{D t}=-\frac{1}{\rho} \nabla p+\mathbf{g}, \\
& \frac{D \rho}{D t}=-\rho \nabla \cdot \mathbf{v}, \\
& \frac{D p}{D t}=c_{s}^{2} \frac{D \rho}{D t},
\end{aligned}
$$

where $D / D t$ is the Lagrangian time derivative, $\mathbf{v}$ is the velocity vector, $t$ is time, $\rho$ is density, $p$ is pressure, $\mathbf{g}$ is the vector of the gravitational acceleration, and $c_{s}$ is the speed of sound.

We linearize the velocity, density, and pressure into background and perturbed components:

$$
\begin{aligned}
(u, v, w) & =\left(U+u_{1}, V+v_{1}, 0+w_{1}\right), \\
\rho & =\rho_{0}+\rho_{1}, \\
p & =p_{0}+p_{1},
\end{aligned}
$$

where $(U, V, 0)$ represent horizontal background wind velocities, $\left(u_{1}, v_{1}, w_{1}\right)$ are the perturbed velocities due to internal waves, $\rho_{0}$ and $\rho_{1}$ are the background density and density perturbation, respectively, and $p_{0}$ and $p_{1}$ are the background pressure and pressure perturbation, respectively.

We introduce a new set of variables that includes a factor accounting for the altitude-dependent density and use a hat to denote rescaled variables:

$$
\begin{gathered}
\left(\hat{u}_{1}, \hat{v}_{1}, \hat{w}_{1}\right)=\rho_{0}^{1 / 2}\left(u_{1}, v_{1}, w_{1}\right), \\
\left(\hat{p}_{1}, \hat{\rho}_{1}\right)=\rho_{0}^{-1 / 2}\left(p_{1}, \rho_{1}\right) .
\end{gathered}
$$

We define a causal Laplace transform in which $s$ is the Laplace variable. The initial conditions are that all perturbations vanish. Taking the Fourier-Laplace transform of the original equations produces

$$
\mathbf{A}\left\{\hat{u}_{1}, \hat{v}_{1}, \hat{w}_{1}, \hat{p}_{1}, \hat{\rho}_{1}\right\}^{\mathrm{T}}=0,
$$

where

$$
\mathbf{A}=\left[\begin{array}{ccccc}
\Omega & 0 & U_{z} & i k & 0 \\
0 & \Omega & V_{z} & i l & 0 \\
0 & 0 & \Omega & \partial_{z}-\frac{1}{2 H} & g \\
i k & i l & \partial_{z}-\frac{1}{2 H} & 0 & \Omega \\
0 & 0 & -g+\frac{c_{s}^{2}}{H} & \Omega & -c_{s}^{2} \Omega
\end{array}\right],
$$

where $\left(U_{z}, V_{z}\right)$ are the vertical shears of $(U, V)$, and $H$ is the density scale height,

$$
H=-\rho_{0} / \rho_{0 z},
$$

and $\Omega=s+i k U+i l V$ is the intrinsic frequency relative to the flow induced by the horizontal wind (which corresponds to a singularity of the Laplace transform of the solution), with $(k, l)$ the wavenumbers in the $(x, y)$ directions.

We can eliminate $\hat{u}_{1}, \hat{v}_{1}$, and $\hat{\rho}_{1}$ and write (9) in terms of $\hat{w}_{1}$ and $\hat{p}_{1}$ :

$$
\begin{aligned}
\partial_{z} \hat{w}_{1}= & {\left[\frac{i}{\Omega}\left(k U_{z}+l V_{z}\right)+\frac{g}{c_{s}^{2}}-\frac{1}{2 H}\right] \hat{w}_{1} } \\
& +\left[-\frac{1}{\Omega}\left(k^{2}+l^{2}\right)-\frac{\Omega}{c_{s}^{2}}\right] \hat{p}_{1}, \\
\partial_{z} \hat{p}_{1}= & {\left[\frac{1}{\Omega}\left(-N^{2}-\Omega^{2}\right)\right] \hat{w}_{1}+\left[\frac{1}{2 H}-\frac{g}{c_{s}^{2}}\right] \hat{p}_{1}, }
\end{aligned}
$$


where $N$ is buoyancy frequency

$$
N^{2}=-g \frac{\rho_{0 z}}{\rho_{0}}-\frac{g^{2}}{c_{s}^{2}} .
$$

We define $\zeta_{1}$ as the perturbed vertical displacement with $D \zeta_{1} / D t=w_{1}$. Following (7), we introduce the rescaled perturbed vertical displacement $\hat{\zeta}_{1}=\rho_{0}^{1 / 2} \zeta_{1}$.

Hence we can express (11) and (12) in terms of $\hat{\zeta}_{1}$ and $\hat{p}_{1}$ to obtain two coupled ODEs in $z$ :

$$
\left\{\begin{array}{l}
\partial_{z} \hat{\zeta}_{1} \\
\partial_{z} \hat{p}_{1}
\end{array}\right\}=\mathbf{B} \cdot\left\{\begin{array}{l}
\hat{\zeta}_{1} \\
\hat{p}_{1}
\end{array}\right\},
$$

where

$$
\mathbf{B}=\left[\begin{array}{cc}
-\frac{1}{2 H}+\frac{g}{c_{s}^{2}} & -\frac{k^{2}+l^{2}}{\Omega^{2}}-\frac{1}{c_{s}^{2}} \\
-N^{2}-\Omega^{2} & \frac{1}{2 H}-\frac{g}{c_{s}^{2}}
\end{array}\right] .
$$

Wei et al. (2015) described an anelastic and a compressible formulation in their sections 2 and 3, respectively. After a Fourier-Laplace transform, their (26) for the compressible model yields exactly our (14). An anelastic formulation can be recovered from (14) by setting $c_{s} \rightarrow \infty$. The formal conditions for the validity of this limit are $g H \ll c_{s}^{2}$ and $g L \ll c_{s}^{2}$, where $L$ is the horizontal length scale of motion. However, this anelastic limit is not the same as Wei et al.'s (2015) anelastic model (4). One can check this by comparing their (4) and (26).

In our formulation, the matrix B in (14) reduces to its anelastic form $\mathbf{B}^{\prime}$ :

$$
\mathbf{B}^{\prime}=\left[\begin{array}{cc}
-\frac{1}{2 H} & -\frac{k^{2}+l^{2}}{\Omega^{2}} \\
-N^{2}-\Omega^{2} & \frac{1}{2 H}
\end{array}\right] .
$$

As a result, our Eq. (14) for the anelastic model has two sign differences compared with Wei et al.'s (2015) (4), which should be

$$
\begin{aligned}
\left(D_{t}^{2}+N^{2}\right) \hat{\zeta} & =\left(-\partial_{z}+\frac{1}{2 H}\right) \hat{p} \quad \text { and } \\
\partial_{x}^{2} \hat{p} & =D_{t}^{2}\left(\partial_{z}+\frac{1}{2 H}\right) \hat{\zeta}
\end{aligned}
$$

Following Wei et al.'s (2015) derivation in (5)-(BC4), the analytical solution to our (14) is as in their (9)-(12), but the parameter $A_{i}(k, s)$ in their (11) should have a negative sign. The modified version of Wei et al.'s (2015) (11) should be

$$
A_{i}(k, s)=-\frac{\left(s+i k U_{i}\right)^{2}}{2 H_{i}} .
$$

When $c_{s} \rightarrow \infty$, Wei et al.'s (2015) (30) and (31) now reduce to their (10) and (11), respectively, with the modified $A_{i}(k, s)$ defined above. This difference is due to the fact that the anelastic formulation modifies the thermodynamic relation, as discussed in Bannon (1996). The difference, however, is small and has no impact on the following results. In particular the dispersion relation for waves in an isothermal atmosphere is the same.

\section{Numerical method}

Wei et al. (2015) present an analytical study of the two-dimensional tsunami-induced acoustic-gravity waves in a layered atmosphere. Within each layer, air is uniformly stratified and the wind velocity is a constant. The tsunami is simplified as a traveling Gaussian bump along the sea surface. They consider an anelastic and a compressible model and include the effect of time. They solve the Fourier-Laplace-transformed problem explicitly to obtain an analytical solution to $\hat{\zeta}_{1}$, then perform the inverse transforms to recover the solutions in physical space and in time. As a result, they observe significant wave reflection at the layer boundaries, and find that the acoustic signal is the first to arrive at the ionosphere.

However, in a more realistic atmosphere, because of the vertically varying stratification and background winds, it is not possible to derive an analytic expression for the solution. We solve the Fourier-Laplacetransformed problem numerically to obtain $\hat{\zeta}_{1}$ from (14), where the $z$-dependent parameters are buoyancy frequency $N$, density scale height $H$, and background wind velocities $(U, V)$. Following Wu et al. (2016a), we select values of wavenumber $k$ based on the horizontal dimension and the desired resolution, and select values of Laplace parameter $s$ based on the time range and temporal resolution of interest. For each discrete set of $k$ and $s$, the wave problem becomes a set of second-order ODEs in the vertical given by (14).

The upper radiation boundary conditions used in the steady problems as in Broutman et al. (2014), Wu et al. (2016a,b) do not apply here since there is no response at $t=0$ as the system is causal. Above the domain, we assume that $\hat{\zeta}_{1}$ decays exponentially as altitude increases with a factor of $\lambda^{-}$, where $\lambda^{+}$and $\lambda^{-}$are the two eigenvalues of matrix $\mathbf{B}$ in (14) with positive and negative real parts, respectively. We integrate the vertical ODEs (14) from top to bottom with the upper boundary conditions that $\left\{\hat{\zeta}_{1}, \hat{p}_{1}\right\}^{\mathrm{T}}$ equals the eigenvector corresponding to $\lambda^{-}$. Then at sea level, $\hat{\zeta}_{1}$ is rescaled to match the 
displacement of the tsunami-perturbed lower boundary while the effect of acoustic-gravity waves on the tsunami itself is neglected.

Finally, we recover the solutions by performing an inverse Fourier transform in the horizontal and an inverse Laplace transform in time. We adopt Iseger's algorithm for the inverse Laplace transform. It is fast and accurate: den Iseger (2006) presents a clear discussion of this, noting that full double precision accuracy can be reached in most cases and validating the results for known transform pairs. Iseger's algorithm also has a version that is designed specifically for functions with discontinuities and singularities with the same accuracy. This is useful in dealing with the two-stage bottom boundary conditions in our tsunami profile (15) but is more time-consuming. The results we present use the regular Iseger algorithm for a balance of accuracy and efficiency. Wei et al. (2015) state that their inverse Laplace transform is computationally expensive. We tested the computational time of inverse Laplace transform using Talbot's algorithm (Talbot 1979) and Euler's algorithm (Abate and Whitt 2006) to compare to Iseger's algorithm (den Iseger 2006). The function tested is the two-stage tsunami profile in Wei et al. (2015, their appendix A), with $\hat{f}(k)=1$ and a discontinuity of gradient at $t=\tau=5 \mathrm{~s}$. It is found that Talbot's algorithm cannot recover the two-stage profile in time, while Euler's and Iseger's algorithms perform well for functions with a discontinuity. In terms of efficiency, Iseger's algorithm saves up to $93 \%$ of the running time compared to the other two.

In our case, the full numerical algorithm consists of essentially three parts: solving the vertical ODEs (14) for each Fourier-Laplace component, inverse Fourier transforming to recover solutions in physical space, and inverse Laplace transforming to recover solutions in time. Since we improved the efficiency of inverse Laplace transform by employing den Iseger's algorithm, the most time-consuming part becomes solving the vertical ODEs.

\section{Results}

\section{a. Case 1: Two-layer atmosphere with a Gaussian-shaped tsunami profile}

We consider the case in Wei et al. (2015) and examine propagation through a two-layer atmosphere with a tropopause at $10-\mathrm{km}$ altitude: in the lower layer, buoyancy frequency $N_{1}=0.01 \mathrm{~s}^{-1}$ and density scale height $H_{1}=9 \mathrm{~km}$; in the upper layer, $N_{2}=0.02 \mathrm{~s}^{-1}$ and $H_{2}=6 \mathrm{~km}$. In the anelastic case, the sound speeds $c_{1}$ and $c_{2}$ in the two layers are set to infinity, while in the compressible case, $c_{1}=312 \mathrm{~m} \mathrm{~s}^{-1}$ and $c_{2}=279 \mathrm{~m} \mathrm{~s}^{-1}$ are calculated from $N_{1}, H_{1}$ and $N_{2}, H_{2}$ according to (10) and (13), respectively.

For the bottom boundary, we use the same tsunami profile as in Wei et al. (2015). This has two stages in time: when $t \leq \tau$, the vertical displacement grows linearly from 0 to $1 \mathrm{~m}$ with a structure $f(x)$. Subsequently it keeps its shape but travels to the positive $x$ axis at a constant phase speed $c=200 \mathrm{~m} \mathrm{~s}^{-1}$ :

$$
h(x, t)=\left\{\begin{array}{ll}
(t / \tau) f(x), & 0<t \leq \tau \\
f[x-c(t-\tau)], & t>\tau
\end{array},\right.
$$

where $\tau=1 \min , f(x)=f_{1}(x)=\exp \left(-x^{2} / 2 \sigma_{1}^{2}\right)$, and $\sigma_{1}=$ $20 \mathrm{~km}$. We use the value $\tau=1 \mathrm{~min}$, since our goal is to compare analytic and numerical results rather than realism. (Wei et al. 2015, used $5 \mathrm{~s}$, although this is not mentioned in the paper.)

In Fig. 1, the top panels show the snapshots of the rescaled vertical displacement $\hat{\zeta}_{1}$ in the anelastic case, which is to be compared with Fig. 1 in Wei et al. (2015). We plot the horizontal distribution of $\hat{\zeta}_{1}$ with a magnification factor of 15000 at 0-, 30-, 60-, and 100-km altitudes. The tsunami displacement is also magnified and coincides with the curve at $0 \mathrm{~km}$. The top-left panel is the snapshot at $t=40 \mathrm{~min}$ in a uniformly stratified atmosphere with $N=N_{2}=0.02 \mathrm{~s}^{-1}$ and $H=H_{2}=6 \mathrm{~km}$, while the top-center panel is the snapshot at $t=40 \mathrm{~min}$ but in a two-layer atmosphere with a tropopause at $10-\mathrm{km}$ altitude. Comparing the top-left and top-center panels, we observe smaller displacements at 30-, 60-, and 100-km altitudes in the top-center panel given the same bottom boundary at $0 \mathrm{~km}$. In a two-layer atmosphere, because of the discontinuous stratification, part of the energy carried by gravity waves is reflected downward at the interface, resulting in smaller responses that propagate through the tropopause into the upper layer.

The top-right panel shows the snapshot at $t=5 \mathrm{~min}$ in a two-layer atmosphere. In the anelastic case, internal waves are pure gravity waves. There is no response above $30 \mathrm{~km}$ because the gravity branch has a slower vertical group velocity and has not arrived at higher altitudes at $t=5 \mathrm{~min}$.

The bottom three panels of Fig. 1 are as the top ones but in the compressible case, and they are to be compared to Fig. 5 in Wei et al. (2015). By adding compressibility of the atmosphere, we take into account the acoustic branch of internal waves generated by the tsunami. Comparing the compressible results with the anelastic ones, we observe a response at $60 \mathrm{~km}$ at $t=5 \mathrm{~min}$ in the compressible case, representing the early arrival of acoustic waves. These are emitted by the initial growth of the tsunami disturbance with frequencies higher enough to arrive rapidly at high altitudes (see 


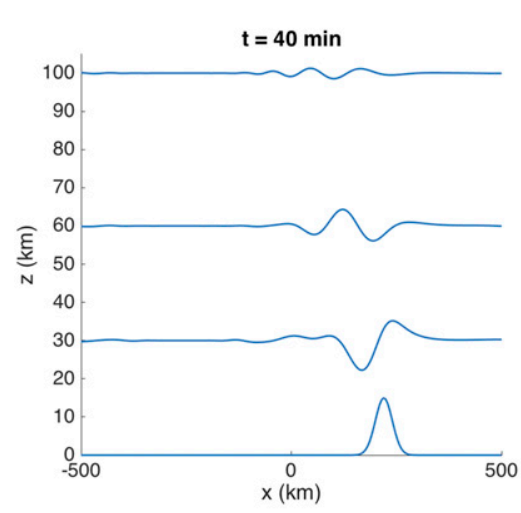

\section{Anelastic case}

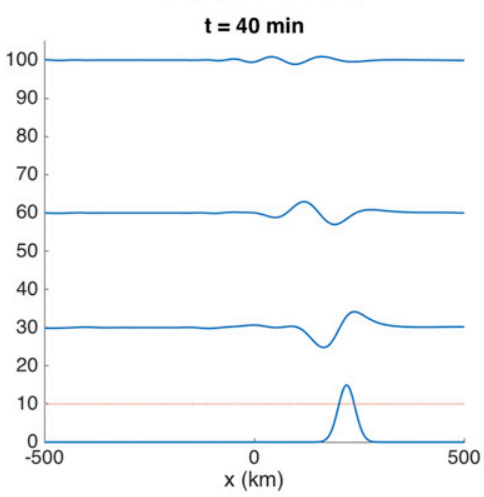

Compressible case
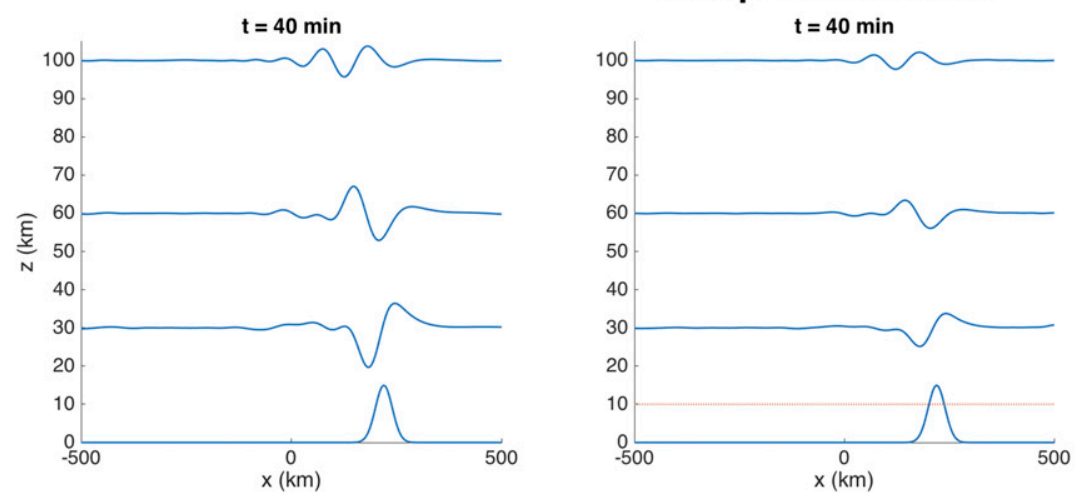
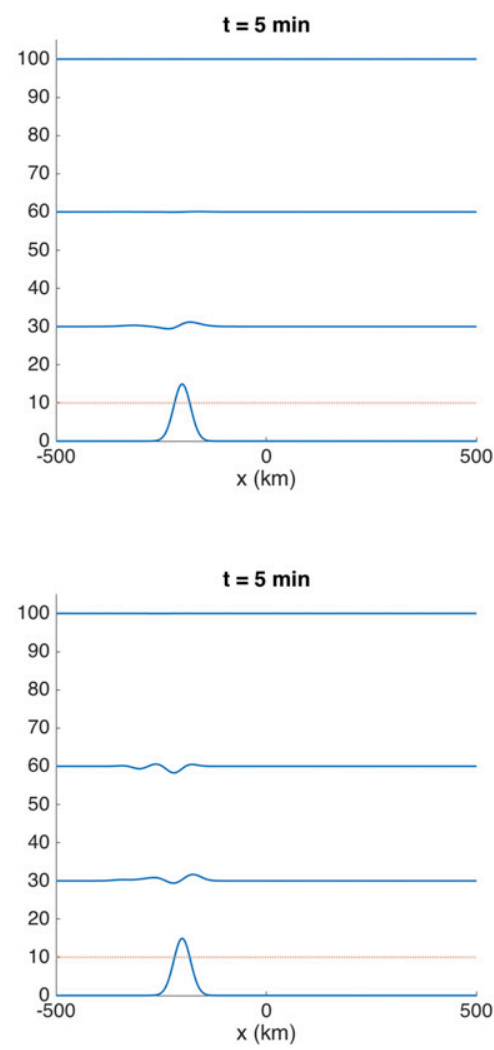

FIG. 1. Snapshots of the rescaled vertical displacement $\hat{\zeta}_{1}$ (with a magnification of 15000 ) at 0-, 30-, 60-, and 100-km altitudes in (top) an anelastic atmosphere and (bottom) a compressible atmosphere a Gaussian-shaped tsunami profile (left) at $t=40 \mathrm{~min}$ in a uniformly stratified atmosphere; (middle) at $t=40 \mathrm{~min}$ in a two-layer atmosphere with a tropopause at $10 \mathrm{~km}$ (red horizontal line), and (right) at $t=5 \mathrm{~min}$ in a two-layer atmosphere. The uniform atmosphere has $N=0.02 \mathrm{~s}^{-1}$ and $H=6 \mathrm{~km}$; the two-layer atmosphere has $N_{\text {lower }}=0.01 \mathrm{~s}^{-1}, H_{\text {lower }}=9 \mathrm{~km}, N_{\text {upper }}=0.02 \mathrm{~s}^{-1}$, and $H_{\text {upper }}=6 \mathrm{~km}$. The tsunami displacement is also magnified and coincides with the curve at $0 \mathrm{~km}$.

Fig. 9.1 of Occhipinti 2016). This process does not occur in the anelastic case.

In Fig. 2, we plot the time evolution of the wavenumberaveraged root-mean-square (RMS) $\hat{\zeta}_{1}$ evaluated at the upper boundary $z=100 \mathrm{~km}$ normalized by the RMS tsunami displacement in a one-atmosphere and a twolayer atmosphere. The anelastic case is in blue and purple, and the compressible case is in orange and yellow. Curves are averaged and smoothed over time using curve fitting among 100 Fourier components with horizontal wavenumber $k$ linearly distributed between $-k_{\max }$ and $k_{\max }$, where $k_{\max }=\pi /$ (the horizontal grid spacing of $12 \mathrm{~km})=2.618 \times 10^{-4} \mathrm{~m}^{-1}$. We also plot Wei's analytical solutions in dashed curves for comparison.

In the anelastic case, the averaged displacement grows slowly from zero in the first $25 \mathrm{~min}$, then increases dramatically, reaching a maximum value of 0.85 and 0.55 within $240 \mathrm{~min}$ in the one- and two-layer atmospheres, respectively. However, in the compressible case, results are very different: there is no signal in the first $6 \mathrm{~min}$, then a sharp rise and drop appear between $t=6$ and 20 min that represent the first arrival of acoustic waves. We estimate the first acoustic signal arrives at $100 \mathrm{~km}$ within approximately $t-\tau=5 \mathrm{~min}$, yielding a vertical group velocity of $333 \mathrm{~m} \mathrm{~s}^{-1}$, which is a reasonable value of sound speed in the atmosphere. Finally, the averaged displacement grows again and reaches its maximum value of 0.88 and 0.42 within $240 \mathrm{~min}$ in the one- and two-layer atmospheres, respectively.

The discrepancies between our Fig. 2 and Wei et al.'s (2015) Fig. 4 are due to the different choices of sound speed. Wei et al. (2015) obtain $c_{1}=319 \mathrm{~m} \mathrm{~s}^{-1}$ and $c_{2}=296 \mathrm{~m} \mathrm{~s}^{-1}$ by choosing a representing value of air temperature in each layer. The problem is that $N, H$, and $c_{s}$ must be related by (13), thus one cannot choose the value of sound speed independently. We fixed this problem in Wei et al. (2015) by calculating $c_{s}$ from $N$ and $H$ using (13), which results in a slightly smaller values of $c_{1}=312 \mathrm{~m} \mathrm{~s}^{-1}$ and $c_{2}=279 \mathrm{~m} \mathrm{~s}^{-1}$ in the compressible case. 


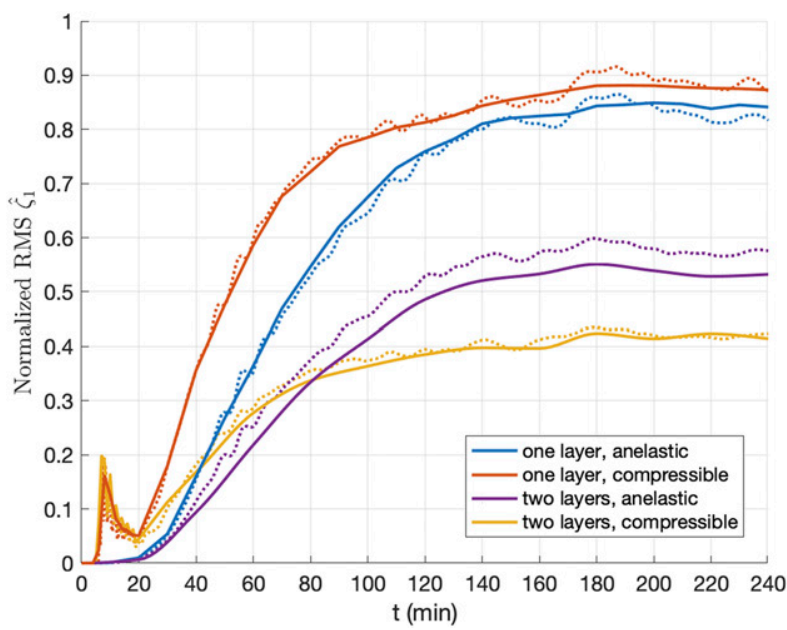

FIG. 2. Time evolution of the normalized root-mean-square (RMS) $\hat{\zeta}_{1}$ at $100 \mathrm{~km}$ in one- and two-layer atmospheres with a Gaussian-shaped tsunami profile. Solid curves show our numerical solution; dashed curves show Wei et al.'s (2015) analytical solution.

By adding compressibility, we find that for short times, the acoustic branch is the first to arrive at the upper atmosphere, thus energy carried by acoustic waves will be rapidly transmitted upward. We conclude that compressibility has a nonnegligible impact on the time evolution of internal waves: during the early stage of wave development, compressibility promotes the speed of energy transmitted through the entire atmosphere.

\section{b. Case 2: Uniformly stratified atmosphere with a jet}

Next, we consider the atmosphere as a uniformly stratified medium with $N=0.02 \mathrm{~s}^{-1}$ and $H=6 \mathrm{~km}$, and use a more realistic tsunami profile as the bottom boundary as a further step toward reproducing realworld conditions. We initially consider an atmosphere at rest. In time, the tsunami displacement has the same growth and traveling stages as in case 1, while in the horizontal we use the model profile of Peltier and Hines (1976),

$$
f=f_{2}(x)=a \operatorname{Ai}(1-X)(X / 2) \exp [(2-X) / 2],
$$

where Ai denotes the Airy function, $X$ is the horizontal coordinate $x$ in units of $100 \mathrm{~km}$, and $a$ is a coefficient that normalizes the maximum $f_{2}|(x)|$ to $0.5 \mathrm{~m}$. The tsunami displacement in the spatial and Fourier domains is plotted in Fig. 3. It has a constant phase speed of $200 \mathrm{~m} \mathrm{~s}^{-1}$ to the left in the traveling stage. The wavenumber corresponding to the maximum vertical displacement is $1.07 \times 10^{-5} \mathrm{~m}^{-1}$. Thus, the dominant intrinsic frequency of the tsunami displacement is $2.14 \times 10^{-3} \mathrm{~s}^{-1}$.

Figure 4 is the snapshot of $\hat{\zeta}_{1}$ with a magnification of 15000 at $t=40 \mathrm{~min}$ through the uniformly stratified
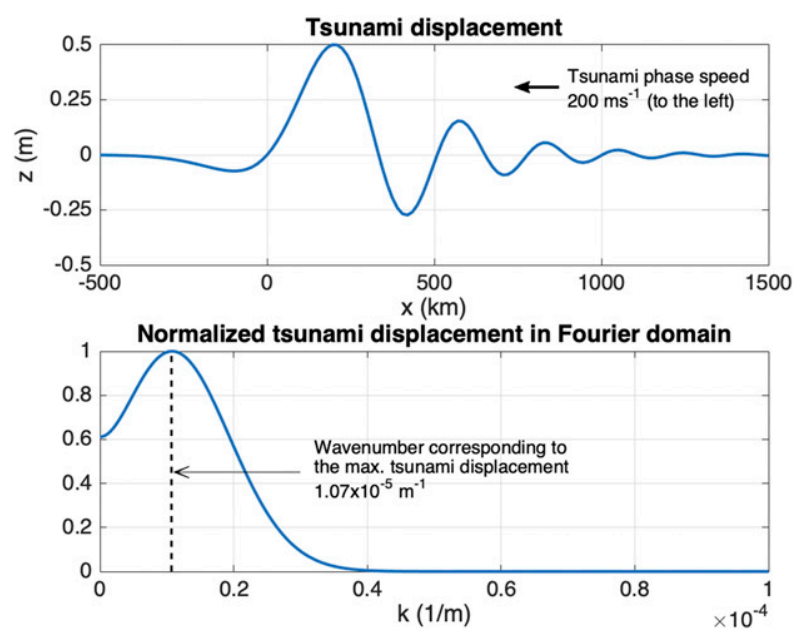

FIG. 3. Model tsunami displacement in (top) spatial and (bottom) Fourier domains from (16).

atmosphere but with the model tsunami profile. Compared to a simple Gaussian bump in the first case, the model tsunami extends up to $1500 \mathrm{~km}$ in the horizontal with a dominant wavelength of approximately $587 \mathrm{~km}$. The left panel is the anelastic case and the right is compressible. We plot wave displacements at 0-, 30-, $60-$, and $100-\mathrm{km}$ altitudes in the windless atmosphere in solid blue curves.

In the left panel, the anelastic case, we track wave crests of the first four leading gravity waves and plot the paths in yellow. In a uniformly stratified medium, paths of constant phase are straight lines. We assume the path corresponding to the gravity wave with horizontal wavenumber $k$ has an angle $\theta$ to the vertical. It should satisfy $N \cos \theta=k c$, where $c$ is the phase speed of the moving topography (Lighthill 2001).

From the tsunami profile in (16) (see also Fig. 3), we find the dominant wavenumber $k_{D}=1.07 \times$ $10^{-5} \mathrm{~m}^{-1}$ and estimate the theoretical value of angle $\theta=\cos ^{-1}\left(k_{D} c / N\right) \approx 84^{\circ}$. On the other hand, our numerical results estimate the angle to be approximately $85^{\circ}$, which agrees well with the theoretical value.

The goal of this case is to examine the effect of horizontal wind jet. We take the jet to be a Gaussian function of altitude:

$$
U(z)=U_{p} \exp \left(-\frac{z-z_{p}}{\sigma_{2}}\right)^{2},
$$

with peak velocity $U_{p}=50 \mathrm{~m} \mathrm{~s}^{-1}$, peak altitude $z_{p}=50 \mathrm{~km}$, and $\sigma_{2}=10 \mathrm{~km}$.

We plot $\hat{\zeta}_{1}$ in a wind jet in dash-dotted purple curves and the wind velocity in dashed red on the left (not to scale). Wave displacements in a wind jet have the same 

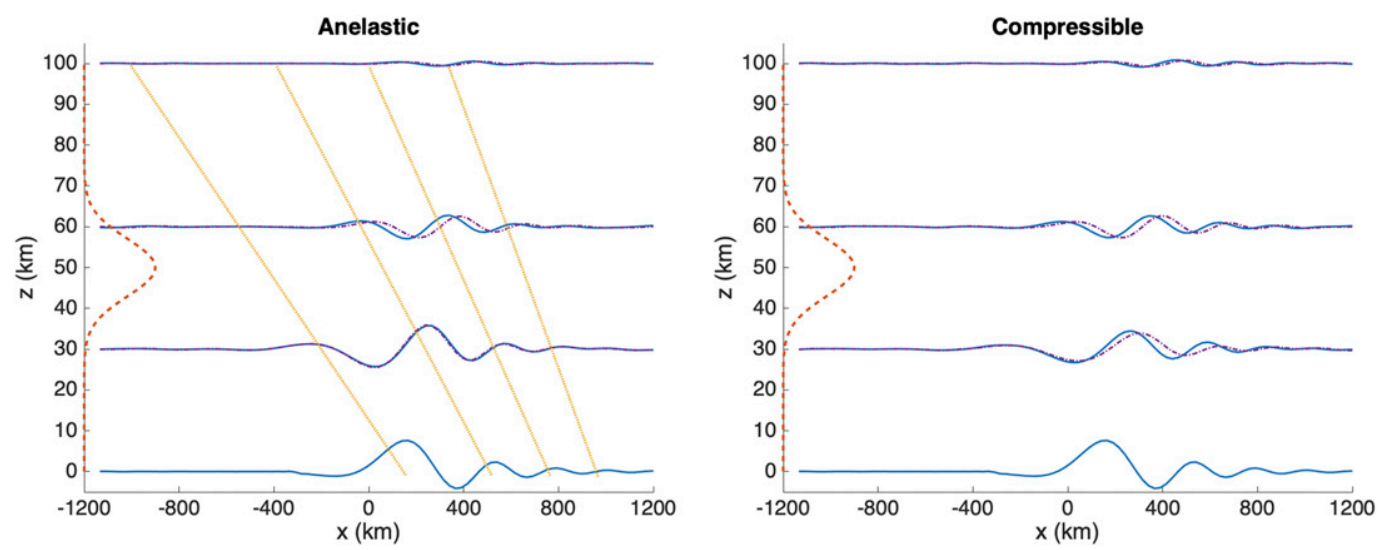

FIG. 4. Snapshots of $\hat{\zeta}_{1}$ (with a magnification of 15000 ) at $0-, 30-, 60-$, and $100-\mathrm{km}$ altitudes at $t=40 \mathrm{~min}$ in a uniformly stratified atmosphere with a model tsunami profile. The blue solid line is in a windless atmosphere, the purple dash-dotted line is in a wind jet, the yellow lines indicate paths of constant phase of pure gravity waves, and the red dashed lines are horizontal wind velocity (not to scale). (left) The anelastic case; (right) the compressible case. The uniform atmosphere has $N=0.02 \mathrm{~s}^{-1}$ and $H=6 \mathrm{~km}$. The tsunami displacement is also magnified and coincides with the curve at $0 \mathrm{~km}$.

order of magnitudes as those in a windless atmosphere. In the anelastic case, there is an obvious shift of wave packets toward the wind direction at $60-\mathrm{km}$ altitude within the jet, while in the compressible case, shifts appear at $30-$ and $60-\mathrm{km}$ altitudes. We conclude that background winds drive wave packets toward the wind direction, thus promoting wave advection in the horizontal, as one might expect.

Figure 5 shows the time evolution of the normalized RMS $\hat{\zeta}_{1}$ at $100 \mathrm{~km}$ as Fig. 2 but with the model tsunami profile. Curves are averaged and smoothed over time using curve fitting among 100 Fourier components with horizontal wavenumber $k$ linearly distributed between $-k_{\max }$ and $k_{\max }$, where $k_{\max }=\pi /$ (the horizontal grid spacing of $30 \mathrm{~km})=1.047 \times 10^{-4} \mathrm{~m}^{-1}$. Blue shows the anelastic case and orange shows the compressible case. Dotted curves show analytical solutions in a windless atmosphere, solid curves show numerical results, and dashed curves show numerical results but in a wind jet.

In the anelastic case, the averaged displacements grows slowly with an increasing slope in the first $65 \mathrm{~min}$, then increases dramatically reaching a maximum value of 0.88 at $t=240 \mathrm{~min}$ in the windless case and 0.85 in a wind jet, respectively. In the compressible case, the first arrival of the fast acoustic branch is observed at $t=6 \mathrm{~min}$ followed by a rapid growth and subsequent decrease between $t=6$ and $20 \mathrm{~min}$. Afterward, the averaged displacements increase and reach their maximum values of 0.88 at $t=240 \mathrm{~min}$ and 0.71 , respectively. The compressible analytical curve captures finescale oscillations over time that Wei et al. (2015) has missed. These oscillations are pronounced for the case with the jet, and presumably are related to modes trapped in the jet.
Comparing results in a windless atmosphere and in a wind jet, we find that background winds result in a smaller response at the upper atmosphere in general. Internal waves are subject to reflection when encountering winds, resulting in less energy that can tunnel through the entire atmosphere. Comparing the anelastic and compressible results, we conclude that compressibility leads to a dramatic response of acoustic waves for short times.

We then vary $\tau$, the duration of the first/linearly growing stage of the tsunami, to investigate its effect

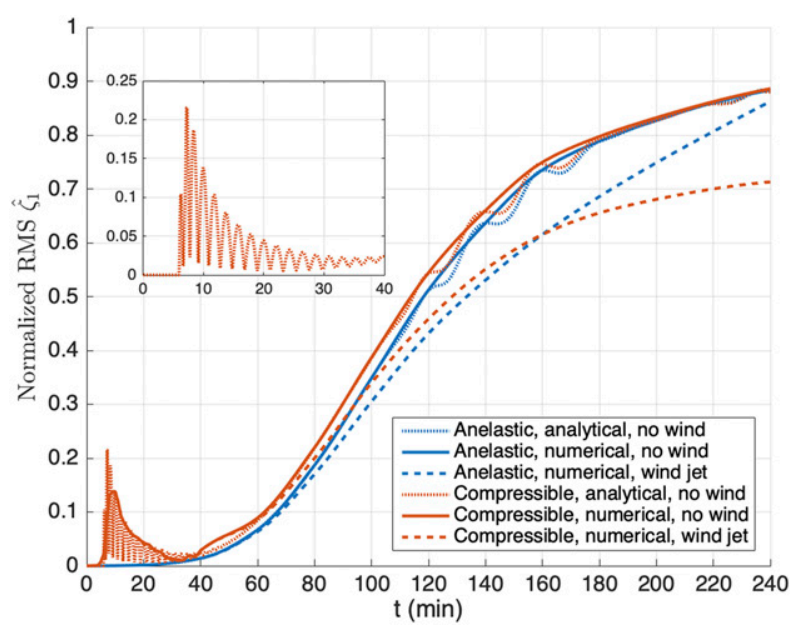

FIG. 5. Time evolution of the normalized RMS $\hat{\zeta}_{1}$ at $100 \mathrm{~km}$ in a uniformly stratified atmosphere with a model tsunami profile. The curves represent the anelastic case (blue) and the compressible case (orange) for analytical results in a windless atmosphere (dotted), numerical results in a windless atmosphere (solid), and numerical results in a wind jet (dashed). 
on the amplitude of the acoustic waves reaching the upper boundary in the first $20 \mathrm{~min}$. In Fig. 6 we find that a fast growth of the first stage of the forcing at the bottom boundary (the tsunami) gives to strong responses at the upper boundary, while a slow disturbance only results in mild responses. This suggests that the response is sensitive to the time scale of the original tsunami displacement, and hence that the initial transient reaching the upper atmosphere will not be as strong if the initial tsunami growth is of the order of minutes (which is unlikely to the case, however).

\section{c. Case 3: A realistic atmosphere with a model tsunami profile}

Finally, we examine propagation through a realistic atmosphere associated with the 2004 Sumatra tsunami with the model tsunami profile as in case 2 . The horizontal wind velocity $U(z)$ is from Drob et al. (2008). The buoyancy frequency $N(z)$ and density scale height $H(z)$ are obtained from the empirical model in Picone et al. (2002). The sound speed $c_{s}(z)$ is calculated from $N$ and $H$ using (10) and (13). Details of the data are described in Broutman et al. (2014) and Wu et al. (2016a). Figure 7 shows the atmospheric parameters as functions of altitude up to $100 \mathrm{~km}$.

In Fig. 8, we take two snapshots at $t=40$ and $80 \mathrm{~min}$, and plot $\hat{\zeta}_{1}$ in the left and right panels, respectively. Comparing the two snapshots, we observe smaller amplitudes in the left panel compared to those in the right panel, indicating that at $t=40 \mathrm{~min}$, energy has partially reached $100 \mathrm{~km}$ but not fully developed yet. From the numerical results, we find wave packets shift to the left by around $480 \mathrm{~km}$ during the $40 \mathrm{~min}$ between the two snapshots, yielding a speed of bottom disturbance of $200 \mathrm{~m} \mathrm{~s}^{-1}$, consistent with the motion of the tsunami.

In Fig. 9, we plot the time evolution of the normalized RMS $\hat{\zeta}_{1}$ at $100 \mathrm{~km}$ in the tsunami case. The blue and orange curves show the anelastic case and compressible case, respectively. In the compressible case, we observe the first signal at $t=5 \mathrm{~min}$ corresponding to the fast acoustic branch. By adding compressibility, the averaged displacement at $240 \mathrm{~min}$ decreases from approximately 0.68 to 0.38 , that is, a $44 \%$ reduction over long times. Compared to the case of the Gaussian jet, the displacement from around 0.85 to 0.68 in the anelastic case and from 0.71 to 0.38 in the compressible case. This are significant reductions of $20 \%$ and $46 \%$, respectively. In addition, we do not see the "ringing" present in the first 40 min of the jet case (see the inset of Fig. 5). This is presumably possible in that case because of the simple jet structure but suppressed in the realistic case.

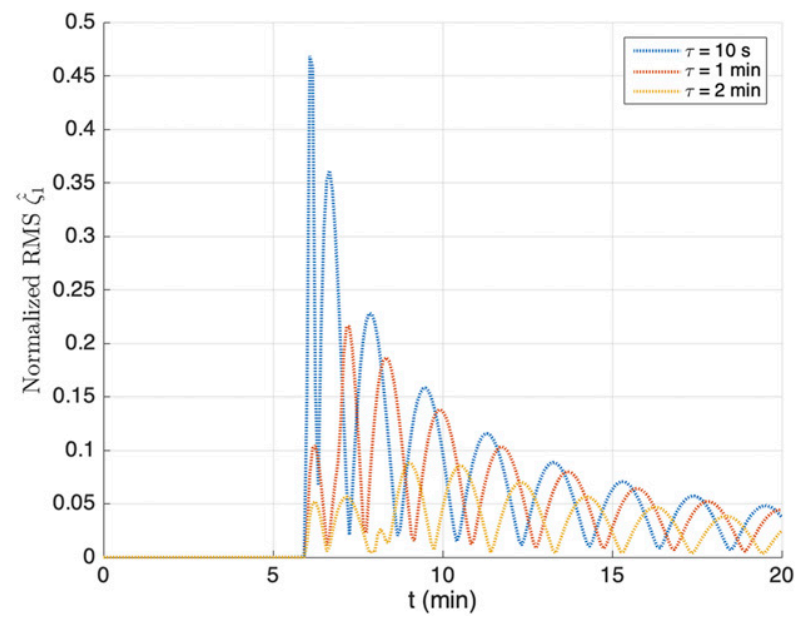

FIG. 6 . The effect of varying $\tau$ on the normalized RMS $\hat{\zeta}_{1}$ in case 2 (compressible, windless).

\section{Conclusions}

We have simulated the time-dependent propagation of tsunami-generated linear internal waves and examined three cases: a two-layer atmosphere with a Gaussian-shaped tsunami profile, a uniformly stratified atmosphere with an idealized wind jet and a model tsunami profile, and a realistic atmosphere with a model tsunami profile.

The first case reproduces some of the results of Wei et al. (2015). By comparing wave amplitudes in a uniformly stratified atmosphere and in a two-layer atmosphere with a tropopause, we confirm that the discontinuous stratification results in smaller responses above the tropopause since part of the energy of internal waves is reflected downward at the interface. By adding compressibility of the atmosphere, we observe a clear rise and drop of wave displacement at $100-\mathrm{km}$ altitude within $6 \mathrm{~min}$ after the tsunami takes place, representing the first arrival of fast acoustic waves at the upper atmosphere.

In the second case, we use a model tsunami profile instead of a Gaussian bump as the lower boundary and add a horizontal background wind jet in a uniformly stratified atmosphere. We compare wave propagation in a windless atmosphere and in a wind jet. Snapshots in time show that waves are driven toward the direction of the wind jet, indicating that wave advection is enhanced by background winds. Then we calculate the time evolution of the normalized and horizontally averaged displacements RMS $\hat{\zeta}_{1}$ at the upper boundary. We observe smaller responses over long times if the wind is present. Hence background winds hinder the vertical transmission of wave energy through the whole atmosphere. We also study the effects of compressibility of 

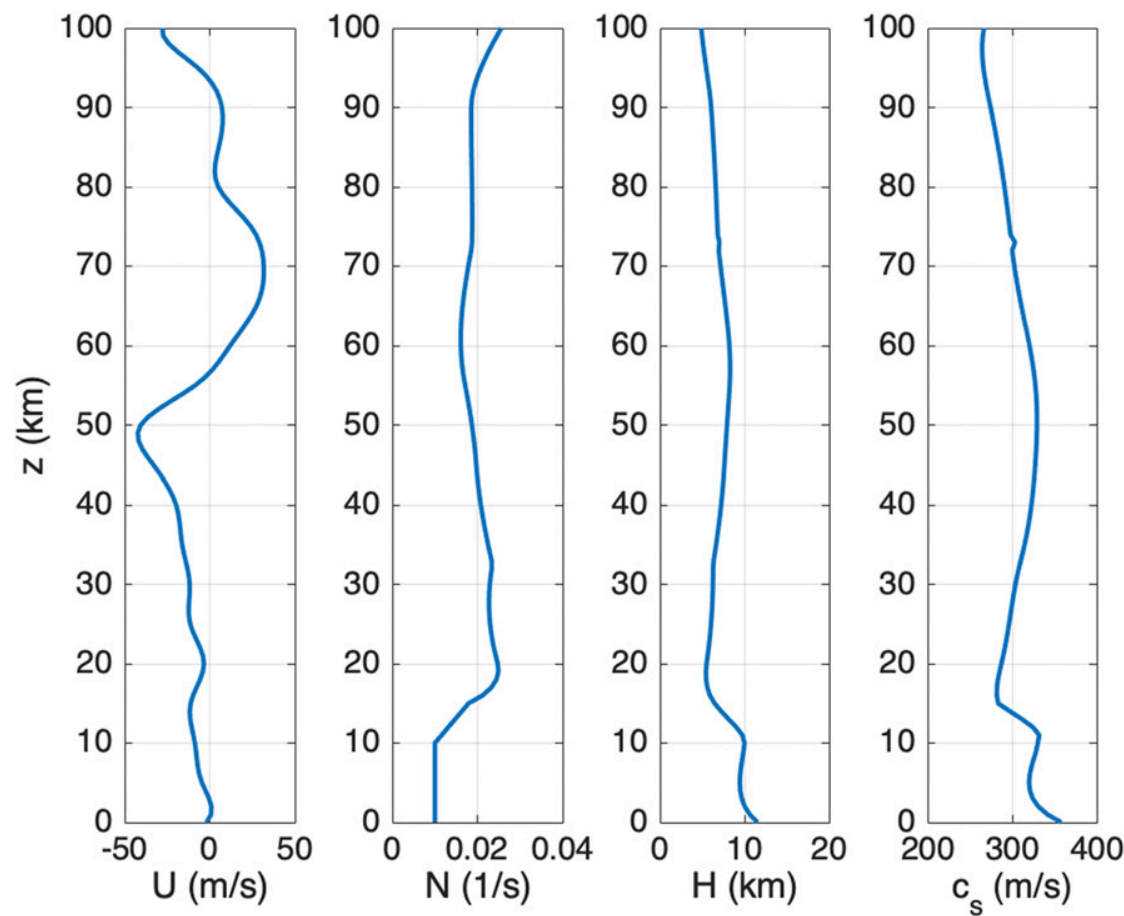

FIG. 7. Atmospheric profile in the 2004 Sumatra tsunami. (left to right) Background wind velocity, buoyancy frequency, density scale height, and speed of sound.

the atmosphere. In a compressible atmosphere, the normalized RMS $\hat{\zeta}_{1}$ shows a sharp rise and drop at the early stage of wave development, and an accumulative growth and level off over long times. We find, in agreement with Wei et al. (2015), that acoustic waves are the first to arrive at the upper atmosphere and have a significant amplitude when compared to the slow gravity waves. In other world, compressibility could promote the timeliness of the tsunami warning system using atmospheric internal waves thanks to the fast acoustic waves.

Finally, we examine wave propagation generated by a model tsunami profile through a realistic atmosphere corresponding to the 2004 Sumatra tsunami. The response of the normalized RMS $\hat{\zeta}_{1}$ at the upper boundary over long times is reduced by approximately $44 \%$ in a compressible atmosphere compared to that in an anelastic atmosphere. We also confirm that the acoustic
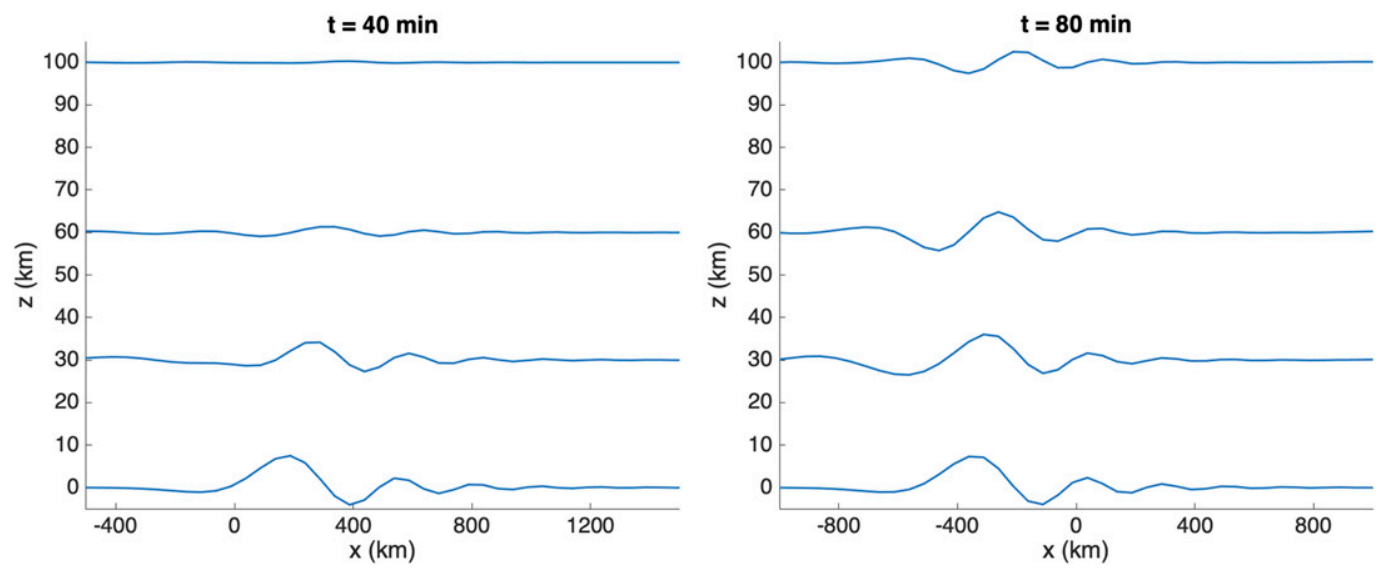

FIG. 8. Snapshots of $\hat{\zeta}_{1}$ (with a magnification of 15000 ) at $0-, 30-, 60-$, and $100-\mathrm{km}$ altitudes in a realistic atmosphere with a model tsunami profile at (left) $t=40$ and (right) $t=80 \mathrm{~min}$. The tsunami displacement is also magnified and coincides with the curve at $0 \mathrm{~km}$. 


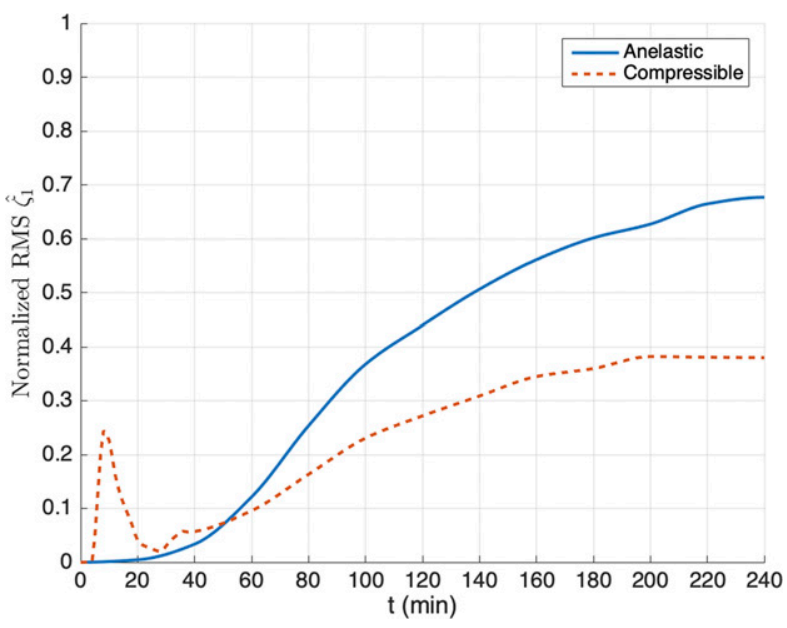

FIG. 9. Time evolution of the normalized RMS $\hat{\zeta}_{1}$ at $100 \mathrm{~km}$ in a realistic atmosphere with a model tsunami profile for the anelastic case (solid) and the compressible case (dashed).

signal is the first to reach the lower ionosphere, as shown for the model and realistic atmospheric profiles as seen in Figs. 5 and 9 (the "spike" visible for early times in the compressible cases).

Wei et al. (2015) examined the initial-value problem for a multilayer atmosphere, where within each layer atmospheric parameters and wind velocities are taken to be constants. Compared to their analytical approach, our numerical approach allows continuous variations of atmospheric parameters and wind velocities in the vertical. The most layers considered by Wei et al. (2015) is 4 , which is clearly inadequate to reproduce the vertical profiles in Fig. 7. In addition, it is known that representing smooth functions by steps in internal wave problems can lead to spurious reflection and transmission (Simanjuntak et al. 2009). Finally, a validated rapid numerical inverse Laplace transform was used.

This research examined the initial-value problem of internal waves excited by tsunamis with the long-term motivation of a potential tsunami early detection system through remote sensing observations of the upper atmosphere. Compared to the works of recent investigators, our numerical method aims to address the challenges of both the computational cost and the complexity of a strongly varying atmosphere during a tsunami. The present approach includes formulating the linear propagation of acoustic-gravity waves through a continuously varying vertical stratification and background wind velocities in the atmosphere, solving the vertical displacement of waves and pressure perturbations numerically as a set of coupled ODEs in the Fourier-Laplace domain, and employing den Iseger's algorithm as a fast and accurate numerical inverse Laplace transform.
It would be of interest to extend our model to include viscosity and attenuation Brissaud et al. (2016), and also to include neutral plasma coupling. This would be particularly desirable in the context of tsunami warning. Further investigation of the first arrival to understand more about its dependence on the initial sea surface displacement and to see whether it can be seen in observations is particularly relevant. Finally, the algorithm we have presented is easily parallelizable, since every wavenumber is independent. This suggests the possibility of examining three-dimensional effects with both $U(z)$ and $V(z)$, extending the results of $\mathrm{Wu}(2018)$.

Acknowledgments. This work was supported by ONR Award N00014-13-1-0347 and by the Department of Mechanical and Aerospace Engineering of University of California San Diego. We thank two anonymous reviewers and Prof. Giovanni Occhipinti for their suggestions that have helped improve the paper substantially.

\section{REFERENCES}

Abate, J., and W. Whitt, 2006: A unified framework for numerically inverting Laplace transforms. INFORMS J. Comput., 18, 408-421, https://doi.org/10.1287/ijoc.1050.0137.

Artru, J., V. Ducic, H. Kanamori, P. Lognonné, and M. Murakami, 2005: Ionospheric detection of gravity waves induced by tsunamis. Geophys. J. Int., 160, 840-848, https://doi.org/10.1111/ j.1365-246X.2005.02552.x.

Bannon, P. R., 1996: On the anelastic approximation for a compressible atmosphere. J. Atmos. Sci., 53, 3618-3628, https://doi.org/10.1175/1520-0469(1996)053<3618:OTAAFA> 2.0.CO;2.

Brissaud, Q., R. F. Garcia, R. Martin, and D. Komatitsch, 2016: Finite-difference numerical modelling of gravitoacoustic wave propagation in a windy and attenuating atmosphere. Geophys. J. Int., 206, 308-327, https://doi.org/10.1093/gji/ggw121.

Broutman, D., S. D. Eckermann, and D. P. Drob, 2014: The partial reflection of tsunami-generated gravity waves. J. Atmos. Sci., 71, 3416-3426, https://doi.org/10.1175/JAS-D-13-0309.1.

den Iseger, P., 2006: Numerical transform inversion using Gaussian quadrature. Probab. Eng. Inform. Sci., 20, 1-44, https://doi.org/ 10.1017/S0269964806060013.

Drob, D. P., and Coauthors, 2008: An empirical model of the Earth's horizontal wind fields: HWM07. J. Geophys. Res., 113, A12304, https://doi.org/10.1029/2008JA013668.

Galvan, D. A., A. Komjathy, M. P. Hickey, and A. J. Mannucci, 2011: The 2009 Samoa and 2010 Chile tsunamis as observed in the ionosphere using GPS total electron content. J. Geophys. Res., 116, A06318, https://doi.org/10.1029/2010JA016204.

,,,--- P. Stephens, J. Snively, Y. T. Song, M. D. Butala, and A. J. Mannucci, 2012: Ionospheric signatures of TohokuOki tsunami of March 11, 2011: Model comparisons near the epicenter. Radio Sci., 47, RS4003, https://doi.org/10.1029/ 2012RS005023.

Godin, O. A., 2014: Dissipation of acoustic-gravity waves: An asymptotic approach. J. Acoust. Soc. Amer., 136, EL411-EL417, https://doi.org/10.1121/1.4902426. 
Hickey, M. P., G. Schubert, and R. L. Walterscheid, 2009: Propagation of tsunami-driven gravity waves into the thermosphere and ionosphere. J. Geophys. Res., 114, A08304, https://doi.org/10.1029/2009JA014105.

,$- \longrightarrow$, and - 2010: Atmospheric airglow fluctuations due to a tsunami-driven gravity wave disturbance. J. Geophys. Res., 115, A06308, https://doi.org/10.1029/2009JA014977.

Laughman, B., D. C. Fritts, and T. S. Lund, 2017: Tsunami-driven gravity waves in the presence of vertically varying background and tidal wind structures. J. Geophys. Res. Atmos., 122, 5076-5096, https://doi.org/10.1002/2016JD025673.

Lighthill, M. J., 2001: Waves in Fluids. 6th ed. Cambridge University Press, $504 \mathrm{pp}$.

Liu, J.-Y., Y.-B. Tsai, K.-F. Ma, Y.-I. Chen, H.-F. Tsai, C.-H. Lin, M. Kamogawa, and C.-P. Lee, 2006: Ionospheric GPS total electron content (TEC) disturbances triggered by the 26 December 2004 Indian Ocean tsunami. J. Geophys. Res., 111, A05303, https://doi.org/10.1029/2005JA011200.

Mai, C.-L., and J.-F. Kiang, 2009: Modeling of ionospheric perturbation by 2004 Sumatra tsunami. Radio Sci., 44, RS3011, https://doi.org/10.1029/2008RS004060.

Makela, J. J., and Coauthors, 2011: Imaging and modeling the ionospheric airglow response over Hawaii to the tsunami generated by the Tohoku earthquake of 11 March 2011. Geophys. Res. Lett., 38, L00G02, https://doi.org/10.1029/ 2011 GL047860.

Occhipinti, G., 2016: The seismology of the planet Mongo: The 2015 ionospheric seismology review. Subduction Dynamics: From Mantle Flow to Mega Disasters, Geophys. Monogr., Vol. 211, Amer. Geophys. Union, 169-182.

—_, P. Lognonné, E. A. Kherani, and H. Hébert, 2006: Threedimensional waveform modeling of ionospheric signature induced by the 2004 Sumatra tsunami. Geophys. Res. Lett., 33, L20104, https://doi.org/10.1029/2006GL026865.

, E. A. Kherani, and P. Lognonné, 2008: Geomagnetic dependence of ionospheric disturbances induced by tsunamigenic internal gravity waves. Geophys. J. Int., 173, 753-765, https://doi.org/10.1111/j.1365-246X.2008.03760.x.

, P. Coïsson, J. J. Makela, S. Allgeyer, A. Kherani, H. Hebert, and P. Lognonné, 2011: Three-dimensional numerical modeling of tsunami-related internal gravity waves in the Hawaiian atmosphere. Earth Planets Space, 63, 847-851, https://doi.org/ 10.5047/eps.2011.06.051.
- L. Rolland, P. Lognonné, and S. Watada, 2013: From Sumatra 2004 to Tohoku-Oki 2011: The systematic GPS detection of the ionospheric signature induced by tsunamigenic earthquakes. J. Geophys. Res. Space Phys., 118, 3626-3636, https://doi.org/10.1002/jgra.50322.

Peltier, W. R., and C. O. Hines, 1976: On the possible detection of tsunamis by a monitoring of the ionosphere. J. Geophys. Res., 81, 1995-2000, https://doi.org/10.1029/JC081i012p01995.

Picone, J. M., A. E. Hedin, D. P. Drob, and A. C. Aikin, 2002: NRLMSISE-00 empirical model of the atmosphere: Statistical comparisons and scientific issues. J. Geophys. Res., 107, 1468, https://doi.org/10.1029/2002JA009430.

Rakoto, V., P. Lognonné, and L. Rolland, 2017: Tsunami modeling with solid Earth-ocean-atmosphere coupled normal modes. Geophys. J. Int., 211, 1119-1138, https://doi.org/ 10.1093/gji/ggx322.

Rolland, L. M., G. Occhipinti, P. Lognonné, and A. Loevenbruck, 2010: Ionospheric gravity waves detected offshore Hawaii after tsunamis. Geophys. Res. Lett., 37, L17101, https://doi.org/ 10.1029/2010GL044479.

Simanjuntak, M. A., J. Imberger, and K. Nakayama, 2009: Effect of stair-step and piecewise linear topography on internal wave propagation in a geophysical flow model. J. Geophys. Res., 114, C12020, https://doi.org/10.1029/2008JC005051.

Talbot, A., 1979: The accurate numerical inversion of Laplace transforms. IMA J. Appl. Math., 23, 97-120, https://doi.org/ 10.1093/imamat/23.1.97.

Wei, C., O. Bühler, and E. G. Tabak, 2015: Evolution of tsunamiinduced internal acoustic-gravity waves. J. Atmos. Sci., 72 , 2303-2317, https://doi.org/10.1175/JAS-D-14-0179.1.

$\mathrm{Wu}$, Y., 2018: The propagation of tsunami-generated internal waves in the atmosphere. Ph.D. thesis, University of California, San Diego, 78 pp.

—, S. G. Llewellyn Smith, J. W. Rottman, D. Broutman, and J.-B. H. Minster, 2016a: The propagation of tsunami-generated acoustic-gravity waves in the atmosphere. J. Atmos. Sci., 73, 3025-3036, https://doi.org/10.1175/JAS-D-15-0255.1.

,,,--- and,$- 2016 \mathrm{~b}$ : The three-dimensional propagation of tsunami-generated internal waves in the atmosphere. Eighth Int. Symp. on Stratified Flows, San Diego, CA, International Association for Hydro-Environment Engineering and Research, https://cpaess.ucar.edu/sites/default/files/meetings/ 2016/issf/papers/wu-yue-article.pdf. 